\title{
Characteristic Analysis of Toxic Traditional Chinese Medicine Contained in Pharmacopoeia 2015
}

\author{
Ming Bai \\ Henan University of traditional Chinese Medicine \\ Henan, China \\ email:1845491137@qq.com
}

Xin Lou

Henan University of traditional Chinese Medicine Henan, China

email:baiming666@126.com

\author{
Yanyan Miao \\ Henan University of traditional Chinese Medicine \\ Henan, China \\ email:13823276201@139.com \\ Mingsan Miao* \\ Henan University of traditional Chinese Medicine \\ Henan, China \\ email:miaomingsan@163.com
}

\begin{abstract}
Toxic Chinese medicine simply refers to the toxic reaction in clinical application of traditional Chinese Medicine, for a long time, The broad masses of the people think in the subconscious is the drug itself has some toxicity, In addition, there are loopholes and misunderstandings in the understanding of traditional Chinese Medicine, In particular, the understanding of toxic traditional Chinese medicine is rare, So far, the concept of toxic traditional Chinese medicine has not been unified, standard, clear provisions, The toxic Chinese medicines were classified in the 2015 edition of the Pharmacopoeia, Among it, "big poison traditional Chinese medicine" has 10 kinds, "toxic traditional Chinese medicine" has 42 kinds, "small poison traditional Chinese medicine" has 31 kinds, However, the pharmacopoeia standard is only the most basic division, With the regularity of drug use and new discovery of disease mechanism, etc., The information of toxic traditional Chinese medicine will be more fully supplemented, Today, this definition of toxic traditional Chinese medicine vague, It has seriously hindered the development of traditional Chinese medicine, the modernization of Chinese medicine, standardization, will also affect the degree of acceptance of Chinese medicine in the world.
\end{abstract}

Keywords: Traditional Chinese medicine; Toxic traditional

\section{Chinese medicine; Pharmacopoeia;}

Traditional Chinese medicine has a long history of use in China, In ancient times, it has contributed greatly to the health of mankind, In recent years, with the development of science and technology and the improvement of people's health awareness, the use of traditional Chinese medicine is more extensive, This is a good trend for the development of traditional Chinese Medicine, But what we can not be ignored is that a very important part of Chinese medicine is composed of toxic traditional Chinese Medicine, According to the records of $<$ Huainan $\mathrm{Zi}$ Xiu Xiu xun>: Shennong well taste the flavor of herbs, water fountain to seek... And in seventy a day. This reminds us that toxic traditional Chinese medicine goes hand in hand, warns us not only to apply traditional Chinese medicine widely, but also to ignore the application of toxic traditional Chinese Medicine, This reminds us that toxic traditional Chinese medicine goes hand in hand, it warns us not only to apply traditional Chinese medicine widely, but also to ignore the application of toxic traditional Chinese Medicine.

TABLE 1 BASIC INFORMATION OF LARGE TOXIN TRADITIONAL CHINESE MEDICINE MARKED IN PHARMACOPOEIA

\begin{tabular}{|c|c|c|c|c|}
\hline $\begin{array}{c}\text { Traditional Chinese } \\
\text { Medicine }\end{array}$ & Dosage & $\begin{array}{l}\text { Processing } \\
\text { Methods }\end{array}$ & $\begin{array}{c}\text { Main Chinese Patent } \\
\text { Medicines }\end{array}$ & Clinical Application \\
\hline monkshood & $3 \sim 9 g$ & squashing & $\begin{array}{l}\text { Xiao Huoluo Dan, Tianhe } \\
\text { Zhuifeng paste }\end{array}$ & $\begin{array}{l}\text { Wind chill, joint pain, cold pain, cold hernia pain, } \\
\text { anaesthesia }\end{array}$ \\
\hline nuces vomicae & $0.3 \sim 0.6 \mathrm{~g}$ & $\begin{array}{l}\text { heated with sand } \\
\text { or oil }\end{array}$ & $\begin{array}{l}\text { ShuFeng DingTong } \\
\text { pill,Shenjin Dan }\end{array}$ & $\begin{array}{l}\text { The throat pain, carbuncle swollen poison, fengbi } \\
\text { pain, fracture }\end{array}$ \\
\hline Prepared nux vomica & $0.3 \sim 0.6 \mathrm{~g}$ & $\begin{array}{l}\text { Crushed into fine } \\
\text { powder }\end{array}$ & JiuFen San & $\begin{array}{l}\text { Traumatic injury, fracture pain, rheumatoid arthritis, } \\
\text { numbness, paralysis, carbuncle sore, sore throat }\end{array}$ \\
\hline henbane & $0.06 \sim 0.6 \mathrm{~g}$ & insolation & $\begin{array}{l}\text { WeiTongning pills, AnChuan } \\
\text { pills }\end{array}$ & $\begin{array}{l}\text { Madness, fengbi Jue pain, cough, stomach pain, } \\
\text { dysentery, prolapse, carbuncle, malign sore embolism }\end{array}$ \\
\hline tiglium & - & $\begin{array}{l}\text { Peeling and } \\
\text { fetching kernel }\end{array}$ & $\begin{array}{l}\text { Fuketongjingwan, } \\
\text { Weichanganwan }\end{array}$ & $\begin{array}{c}\text { Acute abdominal distension pain, blood stasis, phlegm } \\
\text { stasis, diarrhea, edema }\end{array}$ \\
\hline croton cream & $0.1 \sim 0.3 \mathrm{~g}$ & $\begin{array}{l}\text { Frost making } \\
\text { cream }\end{array}$ & $\begin{array}{l}\text { WeiTongDing, } \\
\text { QiZhenwan }\end{array}$ & Abscess abscess, scabies scab, verruca nevus \\
\hline calomel & - & Lapping powder & JiuYiSan & $\begin{array}{l}\text { All the meat inside, dark purple black, carrion to sinus } \\
\text { fistula, pus dripping, durable }\end{array}$ \\
\hline $\begin{array}{l}\text { Rhododendron molle } \\
\text { G.Don. }\end{array}$ & $0.6 \sim 1.5 \mathrm{~g}$ & dry in the sun & $\begin{array}{l}\text { LiuWeimuxiangjiao } \\
\text { Nang, }\end{array}$ & $\begin{array}{l}\text { Rheumatism; headache; swelling or flutter; caries } \\
\text { pain; skin tinea scabies }\end{array}$ \\
\hline radix aconiti agrestis & - & desiccation & $\begin{array}{l}\text { Xiaohuoluodan, } \\
\text { SanQishangyao }\end{array}$ & $\begin{array}{l}\text { Cold and dampness, joint pain, cold pain in the heart, } \\
\text { cold pain and anesthesia hernia }\end{array}$ \\
\hline cantharis & $0.03 \sim 0.06 \mathrm{~g}$ & desiccation & $\begin{array}{l}\text { FuFangbanmaojiao } \\
\text { nang,GanNing Pills }\end{array}$ & $\begin{array}{l}\text { Amenorrhea, tinea, scrofula, wart, ulcer not collapse, } \\
\text { dead inside muscle }\end{array}$ \\
\hline
\end{tabular}


It is not that toxic Chinese medicine has been gradually forgotten or eliminated because of its own toxicity, it is not true, The use of toxic Chinese medicine has also played an important role in the treatment of diseases, Or it can not be said to be irreplaceable. For example, arsenic, As a familiar toxic traditional Chinese Medicine, According to modern studies, it can treat leukemia; Flos daturae also called Datura stramonium, According to the 2015 edition of Pharmacopoeia records, The clinical dosage was between $0.3 \mathrm{~g} \sim 0.6 \mathrm{~g}$, but in the Hua Tuo hemp powder it is the main drug; As far as aconite is concerned, written in Zhang Zhongjing's < Shanghanzabinglun $>$ is used in 20 multi aconite, and the use of Fuzi Lizhong pills has been a good medicine to relieve spleen and stomach deficiency cold. Therefore, it is essential to understand the basic information of toxic traditional Chinese Medicine, Here is a brief from the toxicity of traditional Chinese medicine dosage, processing methods, the main proprietary Chinese medicine and clinical application of four aspects to introduce.

TABLE 2 BASIC INFORMATION OF TOXIC TRADITIONAL CHINESE MEDICINE LABELED IN PHARMACOPOEIA

\begin{tabular}{|c|c|c|c|c|}
\hline $\begin{array}{c}\text { Traditional Chinese } \\
\text { Medicine }\end{array}$ & Dosage & $\begin{array}{l}\text { Processing } \\
\text { Methods }\end{array}$ & $\begin{array}{c}\text { Main Chinese patent } \\
\text { Medicines }\end{array}$ & Clinical Application \\
\hline berberidis & $9 \sim 15 \mathrm{~g}$ & Slice, dry & $\begin{array}{l}\text { JiangTang jiaonang,Xiaozhi } \\
\text { pilula }\end{array}$ & $\begin{array}{c}\text { Dysentery, jaundice, sore throat, red eyes, } \\
\text { traumatic injury }\end{array}$ \\
\hline lacca sinica exsiccata & $2 \sim 5 \mathrm{~g}$ & Setting fire to burn & $\begin{array}{c}\text { TiaoJing HuaYu } \\
\text { pilula,TongJing GanLu pilula }\end{array}$ & $\begin{array}{c}\text { Blood stasis amenorrhea, abdominal masses, insect } \\
\text { abdominal pain }\end{array}$ \\
\hline golden larch bark & - & Shred and dry & $\begin{array}{l}\text { FuFang TuJingPi } \\
\text { spiritus(gelatum) }\end{array}$ & Scabies itch \\
\hline subprostrate sophora & $3 \sim 6 g$ & $\begin{array}{c}\text { Cut thick slices, } \\
\text { dried }\end{array}$ & $\begin{array}{l}\text { KouYanQing pilula,HouTong } \\
\text { tablet }\end{array}$ & $\begin{array}{l}\text { The throat of the moths, sore throat, gingival } \\
\text { swelling and sore throat }\end{array}$ \\
\hline moleplant seed & $1 \sim 2 \mathrm{~g}$ & Break with time & $\begin{array}{l}\text { YuShupowder, ZhouShi } \\
\text { HuiSheng pills }\end{array}$ & $\begin{array}{c}\text { The two barrier, edema, phlegm, stagnation of } \\
\text { blood stasis fullness, amenorrhea; external } \\
\text { treatment of tinea }\end{array}$ \\
\hline $\begin{array}{c}\text { defatted } \\
\text { MOLEPLANT SEED }\end{array}$ & $0.5 \sim 1 \mathrm{~g}$ & $\begin{array}{l}\text { Peeling and } \\
\text { fetching kernel }\end{array}$ & ZiJin Ding,ShiYi JiuJi Pills & The same as above \\
\hline $\begin{array}{l}\text { preparemonkshd } \\
\text { moter root }\end{array}$ & $1.5 \sim 3 \mathrm{~g}$ & Slice, dry & Xiao HuoLuo Dan,MuGua Pills & $\begin{array}{l}\text { Arthralgia due to wind cold dampness, breast pain, } \\
\text { chest pain and joint pain }\end{array}$ \\
\hline rhizoma arisaematis & - & Wash and dry & YuZhen Powder & Carbuncle swelling, snake bug bite \\
\hline $\begin{array}{l}\text { processed Rhizoma } \\
\text { Arisaematis }\end{array}$ & $3 \sim 9 \mathrm{~g}$ & Slice and dry & $\begin{array}{l}\text { WuHu powder,NiuHuang } \\
\text { HuaDu tabelts }\end{array}$ & Carbuncle swelling, snake bug bite \\
\hline semen momordicae & $0.9 \sim 1.2 \mathrm{~g}$ & Mash in use & $\begin{array}{l}\text { WanLing JinGu mastic, XiaoJin } \\
\text { pills }\end{array}$ & $\begin{array}{l}\text { Sores, mastitis, scrofula, Zhilou, stem paralysis, } \\
\text { favus of the scalp }\end{array}$ \\
\hline kanziol & $0.5 \sim 1.5 \mathrm{~g}$ & dry & $\begin{array}{l}\text { ZhouChe pills,Meng Zheng } \\
\text { pills }\end{array}$ & $\begin{array}{l}\text { the phlegm is accumulated, the air is adverse to } \\
\text { cough, and the two are not good, the carbuncle is } \\
\text { swollen and sore }\end{array}$ \\
\hline rhizoma curculiginis & $3 \sim 10 \mathrm{~g}$ & Cut section, drying & $\begin{array}{l}\text { GengNianAn jiaonang,ShenQi } \\
\text { ErXian tabelts }\end{array}$ & $\begin{array}{l}\text { Impotence cold sperm, bones and muscles atrophy } \\
\text { soft, waist Lengtong, Yang deficiency cold diarrhea }\end{array}$ \\
\hline rhizoma typhonii & $3 \sim 6 g$ & - & $\begin{array}{c}\text { ChuangShang } \\
\text { powders,ZhongTongAn } \\
\text { jiaonnag }\end{array}$ & $\begin{array}{l}\text { Eye and mouth breathing oblique, dysphasia, } \\
\text { tetanus, headache, snakebite }\end{array}$ \\
\hline ginkgo & $5 \sim 10 \mathrm{~g}$ & Mash in use & $\begin{array}{c}\text { RuYi DingChuan pills,BaiDai } \\
\text { pills }\end{array}$ & $\begin{array}{l}\text { Phlegm cough, leucorrhea cloudiness, frequent } \\
\text { micturition }\end{array}$ \\
\hline Pinellia ternata & $3 \sim 9 g$ & Mash in use & $\begin{array}{l}\text { ShenSu pills, TongXuan LiFei } \\
\text { pills }\end{array}$ & $\begin{array}{c}\text { Cough and phlegm, phlegm throb, wind phlegm } \\
\text { dizziness, headache, nausea and vomiting, phlegm } \\
\text { syncope }\end{array}$ \\
\hline cinnabar & $0.1 \sim 0.5 \mathrm{~g}$ & dry by airing & $\begin{array}{l}\text { SuHeXiang } \\
\text { pills, AnGong } \\
\text { NiuHuang pills }\end{array}$ & $\begin{array}{c}\text { Palpitations easy surprised, insomnia, epilepsy, } \\
\text { infantile convulsion, blurred vision, stomatitis, } \\
\text { pharyngitis }\end{array}$ \\
\hline $\begin{array}{l}\text { Physochlaina } \\
\text { macrophylla }\end{array}$ & $0.1 \sim 0.2 \mathrm{~g}$ & Mash in use & HuaShanShen tablet & Cold cough insomnia. Mark. \\
\hline scorpio & $3 \sim 6 g$ & Wash and dry & $\begin{array}{l}\text { NiuHuang QianJin powder, } \\
\text { ZhiJing Powder }\end{array}$ & $\begin{array}{l}\text { Spasms, stroke hemiplegia, mouth worm, tetanus, } \\
\text { rheumatoid arthritis, ulcers }\end{array}$ \\
\hline Wu Hua & $1.5 \sim 3 g$ & - & & Scabies carbuncle, frostbite favus of the scalp \\
\hline cocklebur fruit & $3 \sim 10 \mathrm{~g}$ & - & $\begin{array}{l}\text { XinQin granules, BiYuanShu } \\
\text { oral liquid }\end{array}$ & $\begin{array}{c}\text { Cold headache, stuffy nose runny nose, } \\
\text { nasosinusitis }\end{array}$ \\
\hline anemones raddeanae & $1 \sim 3 g$ & Wash and dry & $\begin{array}{l}\text { QianLieTong tablet, ZaiZao } \\
\text { pills }\end{array}$ & $\begin{array}{l}\text { The wind cold dampness, spasm of limbs, joint } \\
\text { pain, carbuncle swollen fester }\end{array}$ \\
\hline monkshood & $3 \sim 15 \mathrm{~g}$ & Direct human drug & $\begin{array}{l}\text { TianMa pills, FuZi LiZhong } \\
\text { pills }\end{array}$ & $\begin{array}{c}\text { Cold vomiting and diarrhea, abdominal cold pain, } \\
\text { kidney yang deficiency, Yinhan edema, Yang } \\
\text { exogenous, hsbt }\end{array}$ \\
\hline azedarach & $3 \sim 6 g$ & Shred and dry & ErTong QingRe DaoZhi pills & Scabies itch \\
\hline multibanded krait & $2 \sim 5 \mathrm{~g}$ & $\begin{array}{l}\text { Ethanol soaking } \\
\text { treatment }\end{array}$ & $\begin{array}{l}\text { TongBi pills, ZhongFeng } \\
\text { HuiChun pills }\end{array}$ & $\begin{array}{l}\text { In rheumatoid arthritis, numbness spasm, eye in the } \\
\text { air whistle oblique, hemiplegia, convulsive spasm, } \\
\text { tetanus, leprosy, scabies }\end{array}$ \\
\hline $\begin{array}{l}\text { Radix Euphorbiae } \\
\text { Pekinensis }\end{array}$ & $1.5 \sim 3 \mathrm{~g}$ & $\begin{array}{l}\text { Cut thick slices, } \\
\text { dried }\end{array}$ & ShiZao pills & $\begin{array}{c}\text { Thoracic and abdominal seeper, phlegm } \\
\text { accumulation, inverse gas cough, two urination, } \\
\text { carbuncle sore }\end{array}$ \\
\hline $\begin{array}{l}\text { prepared kusnezoff } \\
\text { monkshood root }\end{array}$ & $1.5 \sim 3 \mathrm{~g}$ & Slice and dry & SanQi ShangYao pills(capsule) & $\begin{array}{l}\text { Cold and dampness, joint pain, cold pain in the } \\
\text { heart, cold pain and anesthesia hernia }\end{array}$ \\
\hline
\end{tabular}


TABLE 2 BASIC INFORMATION OF TOXIC TRADITIONAL CHINESE MEDICINE LABELED IN PHARMACOPOEIA

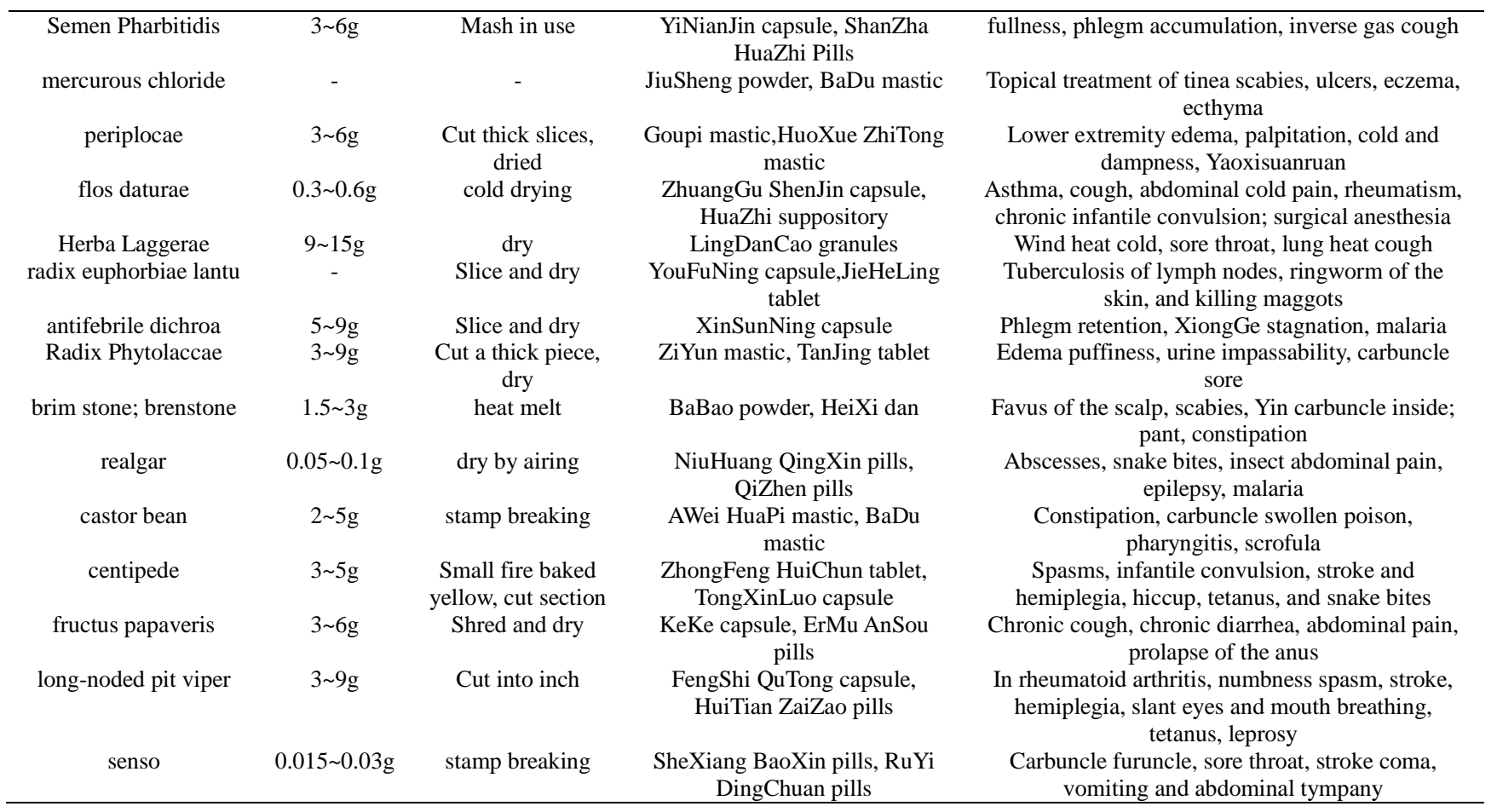

TABLE 3 BASIC INFORMATION OF "SMALL POISON" CHINESE MEDICINE LABELED IN PHARMACOPOEIA

\begin{tabular}{|c|c|c|c|c|}
\hline $\begin{array}{c}\text { Traditional } \\
\text { Chinese Medicine }\end{array}$ & Dosage & $\begin{array}{l}\text { Processing } \\
\text { Methods }\end{array}$ & Main Chinese Patent Medicines & Clinical Application \\
\hline $\begin{array}{l}\text { obtuseleaf erycibe } \\
\text { stem }\end{array}$ & $3 \sim 6 g$ & Slice, dry & $\begin{array}{c}\text { FengLiaoXing FengShi DieDa } \\
\text { YaoJiu }\end{array}$ & Rheumatic pain, hemiplegia, swelling or flutter \\
\hline kamuning & $6 \sim 12 \mathrm{~g}$ & cut up & $\begin{array}{l}\text { TongZhongLing,SanJiu WeiTai } \\
\text { electuary }\end{array}$ & $\begin{array}{l}\text { Stomach pain, rheumatism, toothache, swelling and } \\
\text { pain, snake bite }\end{array}$ \\
\hline cockroach & $3 \sim 10 \mathrm{~g}$ & Sun dried & DaQiLi powder & $\begin{array}{l}\text { Rib fracture, traumatic injury, blood stasis and blood } \\
\text { stasis amenorrhea, postpartum abdominal pain }\end{array}$ \\
\hline $\begin{array}{l}\text { fructus gleditsiae } \\
\text { sinensis }\end{array}$ & $1 \sim 1.5 \mathrm{~g}$ & triturate & $\begin{array}{l}\text { YunXiang QuFeng ZhiTong } \\
\text { tincture, ZhengGu shui }\end{array}$ & $\begin{array}{l}\text { Stroke clenched jaw, remain unconscious, smooth } \\
\text { epilepsy phlegm, closed orifices, stubborn phlegm } \\
\text { cough, expectoration, dry stool }\end{array}$ \\
\hline Chinaberry fruit & $5 \sim 10 \mathrm{~g}$ & triturate & $\begin{array}{l}\text { YiGanNing granules, SanZi } \\
\text { powder }\end{array}$ & $\begin{array}{c}\text { Liver fire, chest pain, abdominal distention, hernia, } \\
\text { insect abdominal pain }\end{array}$ \\
\hline Fructus Podophylli & $3 \sim 9 g$ & dry & ShiYiWei NengXiao pills & $\begin{array}{c}\text { Blood stasis amenorrhea, dystocia, stillbirth, } \\
\text { mazischesis }\end{array}$ \\
\hline hairy euphorbia & $6 \sim 9 \mathrm{~g}$ & Cut section, drying & Ling Yuan WanYing cha & $\begin{array}{c}\text { Furuncle, dysentery, diarrhea, hematuria, eczema, tinea } \\
\text { pedis, skin itching }\end{array}$ \\
\hline bdella & $1 \sim 3 g$ & Cut section, drying & $\begin{array}{l}\text { DaHuang ZheChong pills, } \\
\text { QingNao JiangYa tablet }\end{array}$ & Blood stasis due to stroke, traumatic injury \\
\hline mugwort & $3 \sim 9 g$ & $\begin{array}{l}\text { Remove the stems } \\
\text { and sift the ashes }\end{array}$ & AiFu NuanGong pills & $\begin{array}{l}\text { Vomiting blood, nose bleeding, uterine bleeding, } \\
\text { menorrhagia, fetal bleeding, cold pain in the lower } \\
\text { abdomen, the cold, cold uterus infertility }\end{array}$ \\
\hline $\begin{array}{l}\text { Rhizoma } \\
\text { Menispermi }\end{array}$ & $3 \sim 9 \mathrm{~g}$ & $\begin{array}{l}\text { Cut thick slices, } \\
\text { dried }\end{array}$ & BeiDouGen capsule & Sore throat, heat dysentery, rheumatism \\
\hline anisetree bark & $6 \sim 9 g$ & Break and dry & ShuJin pills, ShanYao pills & Rheumatism, lumbago by \\
\hline knoxia root & $1.5 \sim 3 \mathrm{~g}$ & $\begin{array}{l}\text { Cut thick slices, } \\
\text { dried }\end{array}$ & ZiJin Ding, YuShu powder & $\begin{array}{l}\text { Thoracic and abdominal seeper, phlegm accumulation, } \\
\text { inverse gas cough, two urination, carbuncle sore }\end{array}$ \\
\hline Radix zanthoxyli & $5 \sim 10 \mathrm{~g}$ & $\begin{array}{l}\text { Slice or segment, } \\
\text { dry }\end{array}$ & $\begin{array}{l}\text { ZhengGu Shui, GongYanPing } \\
\text { tablet }\end{array}$ & $\begin{array}{c}\text { Bruises, stomach pain, toothache, rheumatism, snake } \\
\text { bite }\end{array}$ \\
\hline evodia rutaecarpa & $2 \sim 5 g$ & - & AiFu NuanGong pills & $\begin{array}{c}\text { Cold damp beriberi, by the line of abdominal pain, } \\
\text { abdominal distention, acid regurgitation and vomiting, } \\
\text { diarrhoea }\end{array}$ \\
\hline $\begin{array}{c}\text { Picrasma } \\
\text { quassioides }\end{array}$ & $1 \sim 4.5 \mathrm{~g}$ & Slice, dry & $\begin{array}{c}\text { XiaoYan LiDan tablet, FuFang } \\
\text { KuMu XiaoYan tablet }\end{array}$ & $\begin{array}{c}\text { Cough, sore throat, dysentery, eczema, sore furuncle, } \\
\text { snake bites }\end{array}$ \\
\hline $\begin{array}{l}\text { semen armeniacae } \\
\text { amarae }\end{array}$ & $5 \sim 10 \mathrm{~g}$ & pound to pieces & KuXingRen granules & Cough, asthma, chest full of phlegm, constipation \\
\hline $\begin{array}{l}\text { Fohum aconiti } \\
\text { kusnezoffii }\end{array}$ & $1 \sim 1.2 \mathrm{~g}$ & Timely drying & SanQiXueShangNing powder & fever, diarrhea, abdominal pain, headache, toothache \\
\hline
\end{tabular}


TABLE 3 BASIC INFORMATION OF "SMALL POISON" CHINESE MEDICINE LABELED IN PHARMACOPOEIA

\begin{tabular}{|c|c|c|c|c|}
\hline $\begin{array}{l}\text { fructus dauci } \\
\text { carotae }\end{array}$ & $3 \sim 9 g$ & dry & HuaChong pills & $\begin{array}{l}\text { Ascariasis, pinworms, tapeworm disease, insect } \\
\text { abdominal pain, infantile malnutrition }\end{array}$ \\
\hline Java brucea & $0.5 \sim 2 \mathrm{~g}$ & $\begin{array}{l}\text { Husk and } \\
\text { impurities }\end{array}$ & YaDanZiYou Ruan capsule & Dysentery, malaria \\
\hline Paris polyphylla & $3 \sim 9 \mathrm{~g}$ & Slice and dry & $\begin{array}{l}\text { SanQiXueShangNing capsule, } \\
\text { XiaoEr TuiRe admixture }\end{array}$ & $\begin{array}{l}\text { Furuncle and carbuncle, sore throat, snake bites, pain } \\
\text { or flutter, convulsion }\end{array}$ \\
\hline JIXingZI & $3 \sim 5 g$ & Dry and peel & $\begin{array}{c}\text { ShuJin ZhiTong tincture, XiaoYu } \\
\text { DingTong mastic }\end{array}$ & In the lump, amenorrhea, dysphagia \\
\hline fructus cnidii & $3 \sim 10 \mathrm{~g}$ & dry & FuYanPing capsule & $\begin{array}{l}\text { Vulvae leucorrhea, pruritus and eczema, dampness } \\
\text { lumbago, kidney impotence, palace cold infertility }\end{array}$ \\
\hline fructus gleditsiae & $1 \sim 1.5 \mathrm{~g}$ & Dry and mash & $\begin{array}{l}\text { XiaoEr JieRe pills, KaiXiong } \\
\text { ShunQi pills }\end{array}$ & $\begin{array}{c}\text { Stroke clenched jaw, remain unconscious, epilepsy } \\
\text { phlegm, closed orifices, throat phlegm, stubborn } \\
\text { phlegm cough }\end{array}$ \\
\hline $\begin{array}{l}\text { thick wood-fern } \\
\text { rhizome }\end{array}$ & $4.5 \sim 9 \mathrm{~g}$ & $\begin{array}{l}\text { Cut thick slices, } \\
\text { dried }\end{array}$ & KangGan granules & Insect abdominal pain, sore \\
\hline $\begin{array}{l}\text { Dryopteris } \\
\text { crassirhizoma } \\
\text { charcoal }\end{array}$ & $5 \sim 10 \mathrm{~g}$ & dry by airing & FuFang QingDai pills & $\begin{array}{c}\text { Gonorrhea, leucorrhea excessive, rheumatism, joint, } \\
\text { waist and knee pain }\end{array}$ \\
\hline Purple Kei aspidium & $3 \sim 15 \mathrm{~g}$ & $\begin{array}{l}\text { Remove the non } \\
\text { main roots and dry }\end{array}$ & - & fever, dysentery, uterine bleeding \\
\hline Fructus Tribuli & $6 \sim 10 \mathrm{~g}$ & - & $\begin{array}{l}\text { BuYi JiLi pills, JiuWei GanTai } \\
\text { capsule }\end{array}$ & $\begin{array}{l}\text { Headache and dizziness, chest pain, breast milk closed } \\
\text { eyes, ocular itching, rubella }\end{array}$ \\
\hline Entada phaseoloides & $10 \sim 15 \mathrm{~g}$ & $\begin{array}{l}\text { Shelled fry, } \\
\text { levigation }\end{array}$ & QiWei KeTengZi pills & Pale, limb weakness, abdominal pain, poor appetite \\
\hline $\begin{array}{l}\text { fructus carpesii } \\
\text { abrotanoidis }\end{array}$ & $3 \sim 9 g$ & dry & HuaJi KouFuYe & $\begin{array}{l}\text { Ascariasis, pinworms, tapeworm disease, insect } \\
\text { abdominal pain, infantile malnutrition }\end{array}$ \\
\hline $\begin{array}{l}\text { hooker winghead } \\
\text { root }\end{array}$ & $1 \sim 3 g$ & Cut section, drying & ShiErWei YiShou powder & $\begin{array}{c}\text { Detoxification, eliminating pathogenic heat, clearing } \\
\text { away heat and stopping dysentery, expelling wind and } \\
\text { smoothing arthralgia }\end{array}$ \\
\hline
\end{tabular}

\section{DISCUSSION}

The above is from four aspects of the 15 edition of the Pharmacopoeia of the toxic traditional Chinese medicine overview. It can be seen from the analysis of 83 toxic traditional Chinese medicine. In terms of dosage, $94 \%$ of the toxicity of traditional Chinese medicine is no more than $10 \mathrm{~g}$, only three needles, aconite, Herba Laggerae, maximum dose of 5 herbs in purple Kei and To Ko can reach $15 \mathrm{~g}$ aspidium; Another arenobufagin, cantharidin, dosage of realgar etc. 11 toxicity of traditional Chinese medicine does not exceed $1 \mathrm{~g}$, The dose of Venenum Bufonis is more in the $0.015 \mathrm{~g}$ to $0.03 \mathrm{~g}$; In the processing of traditional Chinese Medicine, over millennia, Most traditional Chinese medicines should be processed to alleviate some side effects, Ensure medication safety, Give full play to the role of traditional Chinese medicine in the prevention and treatment of diseases, Processing is an indispensable step for the toxicity of traditional Chinese Medicine, For example, containing toxic ingredients Aconitum Caowu, After high temperature treatment, Hydrolysis into small toxic aconitine, To reduce the toxicity of Aconitum Aconitum but does not reduce the efficacy; For the drying of Chinese medicine in this step, dry by airing, dry beside or over a fire, dry in the sun is different.

Toxicity is a basic attribute of traditional Chinese medicine. Therefore, toxic traditional Chinese medicine should be used rationally. It shouldn't be eliminated because it's called a toxic word. It should be treated rationally. We can not overgeneralization. In clinical application, the use of Chinese patent medicines for toxic traditional Chinese medicine is also rare. This shows the importance of toxic traditional Chinese Medicine. With the development of toxic traditional Chinese medicine in recent years, more and more researches have been done, For example, the main toxic components, the main toxic target organ, etc., Better use and avoidance of these toxicities, It will play a greater role in the clinical treatment of diseases.

\section{ACKNOWLEDGEMENTS}

Foundation item: Public welfare industry research projects (201507004-2), National international cooperation base (2016-151)

\section{REFERENCE}

[1] Liang Jiehao. Analysis of the application of poisonous Chinese herbal medicine in Chinese medicine prescription [j]. snake annals, 2016, (02): 154-155 176.

[2] Liu Ling, Qin Chen. Application of dosage of toxic traditional Chinese medicine in traditional Chinese medicine prescription [j]. Chinese medicine guide, 2014, (12): 266-267.

[3] Xia Qing, Zhang Xiaoxin, Xu Kexin, Zhang Wenting, Fan Jiaojiao, Zhao Chongjun, Feng Yaru, Ni Yuanyuan, Ma Zhiqiang, Lin Ruichao. "People's Republic of China Pharmacopeia >2015 collection contains studies of toxic toxicity of traditional Chinese medicine [j]. global Chinese medicine, 2017, (03): 377-384.

[4] Pan Yaozong. Araceae toxic medicine toxicity mechanism of [d]. Nanjing University of Chinese Medicine, 2016.

[5] Wu Zhenfeng, Huang Xiaoying, announced Lu, Wang Yaqi, Hu Pengyi, Wang Fang, Zhu Genhua, Song Minxian, Yang Ming. The management of toxic traditional Chinese medicine and the research progress of [j]. quality control of Chinese herbal medicine, 2015, (19): 2974-2978.

[6] Zhang Xingxing, Li Zegeng. Study on the application and mechanism of toxic traditional Chinese medicine in cancer [j]. Journal of Liaoning University of Traditional Chinese Medicine, 2015, (09): 213-215.

[7] Liu Ling, Qin Chen. Application of dosage of toxic traditional Chinese medicine in traditional Chinese medicine prescription [j]. Chinese medicine guide, 2014, (12): 266-267.

[8] WengCehngGuo, Traditional Chinese medicine characteristics of toxic Chinese medicine [d]. Nanjing University of Chinese 
Medicine, 2014.

[9] Tan Yong, Li Jian, Xiao Cheng, Lu Cheng, Lv Aiping. Among the component compatibility is a magazine, with detoxification of toxic Chinese medicine is an important way to control $[\mathrm{j}]$. Chinese of traditional Chinese medicine and Western medicine 2017 (01): $115-118$.

[10] Zhang Guangping, Ye Feng. Toxicity and toxicity classification of toxic traditional Chinese medicine [j]. World Chinese medicine, 2014, (02): 175-177.

[11] Fan Haizhou. Toxic components and processing principles of commonly used toxic traditional Chinese medicine [j]. Chinese Journal of traditional Chinese medicine, 2014, (09): 1335-1336. 